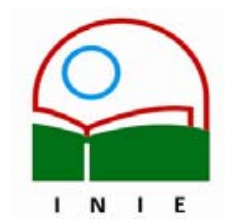

Universidad de Costa Rica

Facultad de Educación

Instituto de Investigación en Educación

ACTUALIDADES INVESTIGATIVAS EN EDUCACION

\title{
INTERVENCIÓN PEDAGÓGICA CON NIÑOS Y NIÑAS MENORES DE TRES AÑOS: EXPERIENCIA EN LA CASA INFANTIL UNIVERSITARIA
}

\author{
PEDAGOGICAL INTERVENTION WITH CHILDREN UNDER THREE YEARS OLD: \\ EXPERIENCE IN THE "CASA INFANTIL" OF THE UNIVERSIDAD DE COSTA RICA.
}

\author{
Jeanneth Cerdas Núñez ${ }^{1}$ \\ Karla Martorell Esquivel ${ }^{2}$
}

\begin{abstract}
Resumen: En este artículo se reflexiona acerca de la importancia de los primeros años de vida en el desarrollo integral del ser humano determinando la importancia de una intervención pedagógica adecuada, para lo cual se comparte la experiencia de la práctica docente con infantes menores de tres años que realizan las estudiantes de la carrera de Bachillerato y Licenciatura en Educación Preescolar de la Universidad de Costa Rica. Se refiere a la experiencia desarrollada durante el año 2005 en la Casa Infantil Universitaria, la cual constituyó según la apreciación de las estudiantes, las familias y el personal del centro, una valiosa oportunidad para favorecer el desarrollo integral de niños y niñas menores de tres años.
\end{abstract}

Palabras Claves: PRIMERA INFANCIA/ EDUCACIÓN TEMPRANA/ FORMACIÓN PROFESIONAL/TAREAS DE DESARROLLO SUBTAREAS DE DESARROLLO/

\begin{abstract}
In this article we seek to meditate about the importance of the first years of life in the human being's integral development determining the importance of an appropriate pedagogic intervention, for which the experience of the educational practice is shared with infants under three years of age, carried out by the students of the career of Preschool Education of the University of Costa Rica. Reference is made specifically to the experience developed during the year 2005 in the Casa Infantil Universitaria, which constituted according to the appreciation of the students, the families and the personnel of the Center, a unvaluable opportunity to favor the integral development of boys and girls under three years of age.
\end{abstract}

Key words: FIRST CHILDHOOD/ EARLY EDUCATION/ PROFESSIONAL FORMATION/ DEVELOPMENT TASKS DEVELOPMENT/ SUBTASKS/

\footnotetext{
1 Máster en Planificación Curricular, Universidad de Costa Rica. Bachiller y Licenciada en Educación Preescolar, Universidad de Costa Rica. Docente de la Escuela de Formación Docente, Universidad de Costa Rica, carrera de Bachillerato y Licenciatura en Educación Preescolar.
}

Correo electrónico: jcerdas@racsa.co.cr

${ }^{2}$ Máster en Psicopedagogía, Universidad Estatal a Distancia. Bachiller y Licenciada en Educación Preescolar, Universidad de Costa Rica. Docente de la Escuela de Formación Docente, Universidad de Costa Rica, carrera de Bachillerato y Licenciatura en Educación Preescolar.

Correo electrónico: karlamartorell@hotmail.com

Artículo recibido: 28 de febrero, 2006

Aprobado: 24 de abril, 2006 


\section{Introducción}

Lo que ocurre durante los primeros años de vida de un ser humano es fundamental tanto para su bienestar inmediato como para su futuro. De esta manera si un niño o niña recibe una atención adecuada desde antes de ser concebido, durante su desarrollo intrauterino y en la primera infancia; en aspectos tales como: salud, nutrición, afecto, estimulación, entre otros, probablemente crecerá sano y tendrá un desarrollo armonioso. "Los primeros años de vida constituyen la etapa más importante del ser humano, caracterizado por el rápido crecimiento físico, mental, social y emocional" (Cerdas, 1995, s.p.)

Es por esta razón que se requiere una atención especial por parte de los adultos que tienen a su cargo a esta población, entre ellos madres y padres de familia, docentes, cuidadores, entre otros, quienes deben esforzarse por brindarles seguridad, confianza y oportunidades para alcanzar su desarrollo.

Es importante mencionar que estos primeros años son críticos para el desarrollo de la inteligencia, el lenguaje, el comportamiento social y la personalidad, de ahí que las experiencias que se le ofrezcan al infante deben procurar estimular todos estos aspectos para favorecer un desarrollo integral.

Según Gálvez (2000) tanto desde el ámbito de la fisiología como el de las ciencias de la salud, la sociología, la psicología y la educación, se pone de manifiesto la importancia de los primeros años de vida no sólo para la formación de la inteligencia, sino para el adecuado desarrollo cognitivo, psicomotor y social de las personas. Durante los dos primeros años de vida se produce la mayor parte del desarrollo de las células neuronales, así como la estructuración de las conexiones nerviosas en el cerebro. En este proceso influyen factores como el estado de salud y la nutrición, pero también la posibilidad de interactuar con el ambiente y con la riqueza y variedad de estímulos disponibles.

Algunas de las condiciones que se le deben brindar a los niños y niñas durante sus primeros años de vida tienen que ir encaminadas hacia la protección contra el peligro físico, una nutrición y atención de la salud adecuadas, una vacunación apropiada, vínculos afectivos saludables, exploración de objetos que les permitan mirar, tocar, escuchar, oler y probar; motivación apropiada para la adquisición y desarrollo del lenguaje, apoyo para 
adquirir nuevas aptitudes motoras, lingüísticas y mentales, la posibilidad de ir obteniendo independencia, apoyo para aprender a controlar su conducta, ocasiones para comenzar a aprender a cuidarse por sí mismos, períodos diarios para jugar con diversos objetos, entre otros.

\section{Incorporación del infante menor de tres años al Sistema Educativo}

En los últimos tiempos, se ha desarrollado una tendencia cada vez más generalizada de que los infantes menores de tres años, participen en alguna experiencia educativa a nivel institucional. Dicha tendencia puede deberse a diferentes factores, que tienen que ver con cambios sociales y de mentalidad, así como con la conciencia creciente de la importancia de la educación en los primeros años.

En este sentido, esta experiencia ha demandado sustituir su naturaleza meramente asistencial por una atención más integral que incorpore el aspecto pedagógico; pues según Feder (2001, pp. 11-12) se ha logrado visualizar que, más allá o más acá de las exigencias sociales por las cuales el infante de edad temprana asiste al Jardín de Niños, hay efectivamente "un niño" o "una niña" y que ese niño o esa niña no sólo requiere cuidados básicos, sino también buenas oportunidades para desarrollar todo su potencial e ingresar en la cultura de una manera activa, creativa y en concordancia con sus necesidades y posibilidades.

Cristina B. de Denies (1992, p. 3) menciona que la educación inicial comprende la educación institucionalizada que se brinda al niño en el ámbito escolar, desde los 45 días de edad hasta el ingreso en la escolaridad primaria obligatoria.

De hecho, el reconocido Informe a la UNESCO de la Comisión Internacional sobre la Educación para el siglo XXI, destaca la importancia de esta etapa para el conjunto de países, proporcionando tres grandes argumentos para promover la expansión de la educación inicial:

Una escolarización iniciada tempranamente puede contribuir a la igualdad de oportunidades al ayudar a superar los obstáculos iniciales de la pobreza o de un entorno social y cultural desfavorecido. Puede facilitar considerablemente la integración escolar de niños procedentes de familias inmigrantes o de minorías culturales y lingüísticas. Además, la existencia de estructuras educativas que acogen a niños en 
edad preescolar facilita la participación de las mujeres en la vida social y económica. (Delors, 1996, p. 133, cit. en Ferrer, 1998, p. 71)

En esta misma línea, las conclusiones de la Conferencia Mundial de Educación para Todos, celebrada en 1990, señalan:

El aprendizaje comienza al nacer. Esto requiere de atención temprana a la infancia y de educación inicial, que se pueden proporcionar a través de disposiciones que impliquen la participación de la familia, las comunidades o programas institucionales, según corresponda. (Delors, 1996, p. 133, cit. en Ferrer, 1998, p. 71, citados en Gálvez 2000).

Es así como en muchos países del mundo han surgido diversas modalidades de atención para esta población. En Costa Rica los niños y niñas menores de tres años están siendo atendidos fundamentalmente por Centros Infantiles de carácter privado, algunas guarderías de instituciones autónomas como RECOPE (Refinadora Costarricense de Petróleo), el ICE (Instituto Costarricense de Electricidad) y el INS (Instituto Nacional de Seguros), así como centros infantiles de instituciones de educación superior de carácter público tales como el Taller Infantil del Instituto Tecnológico de Costa Rica, los Centros Infantiles ubicados en varias sedes de la Universidad de Costa Rica y a partir de setiembre del año 2004, la Casa Infantil Universitaria, también de la Universidad de Costa Rica, ubicada en la Sede Rodrigo Facio.

En cuanto a la Casa Infantil Universitaria (CIU), cabe señalar que es un proyecto de la Vicerrectoría de Vida Estudiantil, que se implementó a partir de setiembre del año 2004 con el objetivo de apoyar a los y las estudiantes para que logren terminar sus estudios, cuando han tenido que abandonar sus carreras universitarias debido a la maternidad o paternidad. En la CIU se atienden a los niños y niñas de estos y estas estudiantes, mientras ellos asisten a clases.

\section{Importancia de la educación en los primeros años de vida}

Como se ha mencionado los primeros años de vida representan una etapa primordial en el proceso de desarrollo y formación de la personalidad. De acuerdo con la Asociación Mundial de Educadores Infantiles, esta afirmación es generalmente aceptada y compartida por psicólogos y pedagogos, independientemente de las tendencias, teorías y escuelas a las que se adscriben; pues todos reconocen la gran plasticidad del cerebro en estas edades, que 
sin constituir una tabla rasa en la que puede inscribirse cualquier impresión, si ofrece amplísimas posibilidades para el establecimiento de conexiones que van a servir de base para el registro y fijación de las más variadas estimulaciones.

Múltiples datos científicos obtenidos en innumerables estudios e investigaciones han evidenciado que en esta etapa existen grandes posibilidades para el desarrollo y la formación de las más diversas capacidades y cualidades personales. Asimismo se reconoce que es aquí donde se sientan las bases y los fundamentos esenciales para todo el desarrollo posterior.

En este sentido la Asociación Mundial de Educadores Infantiles (\$ 27) señala que existen muchas razones para desarrollar experiencias de educación sistematizadas en los primeros años de vida, entre las que recalcan las siguientes:

- Los primeros años de vida, desde el nacimiento hasta los seis años de edad, ponen los cimientos para un crecimiento saludable y armonioso, pues este período está marcado por un rápido crecimiento y por cambios que se ven influenciados por su entorno, dichas influencias pueden ser positivas o negativas, determinando en gran medida cómo será el futuro adulto.

- El cuidado y educación de la niñez mediante una acción integrada adecuada, proporciona un medio para remediar el problema evidente de la desigualdad de oportunidades. Coincidimos en que todos los niños nacen iguales y deben tener igualdad de acceso no sólo al conocimiento y la cultura de todos los pueblos y deben crecer como ciudadanos iguales de su país y del mundo en general.

- La educación en los primeros años complementa al hogar proporcionando la asistencia y formación adecuadas para la promoción del desarrollo total del niño y la niña. Siendo punto de formación no sólo de la niñez, sino también de la familia.

- La igualdad de oportunidades para las mujeres tienen que compaginarse con las necesidades y derechos de los niños y las niñas. Cada vez hay más y más mujeres que trabajan fuera de casa, lo que plantea un problema para la asistencia y educación del infante pequeño.

- La educación de la primera infancia proporciona una valiosa experiencia y preparación para la transición a la escolarización a nivel primario. Los avances de la neurociencia, 
nos demuestran que el sistema nervioso, base y soporte de la personalidad del adulto, se forma en los primeros años.

- No hay segunda oportunidad para la infancia. Por tanto con todos los conocimientos basados en las investigaciones sobre la importancia de esos primeros años de la vida, es fundamental hacer todo lo que sea posible por el bien de cada niño y niña, su salud y nutrición, su crecimiento, aprendizaje y desarrollo, su felicidad.

- El centro educativo representa el principal elemento de socialización para el niño o la niña. Aquí van a aprender a compartir, a esperar y a respetar; hecho, que adquiere una gran relevancia, ya que se presenta como la estructura intermedia entre la propia familia e integración del niño o la niña en los demás estamentos sociales.

- El educador o educadora infantil como profesional conocedor del desarrollo infantil podrá dar seguimiento paso a paso el proceso de desarrollo y maduración de cada niño o niña. Así, puede detectar los posibles desequilibrios, desajustes o déficit que se vayan produciendo. Esta posibilidad de actuación preventiva va a ser determinante y fundamental para el desarrollo de los niños y las niñas.

- La estructura del centro educativo está concebida en función de las necesidades de los niños y las niñas, ofreciendo mayores posibilidades para la manipulación y exploración de los objetos y el espacio. En el marco familiar, los espacios suelen ser limitados, produciéndose continuas prohibiciones, para que no cojan determinados objetos o alcancen o se suban a diversos lugares, hecho que limita sus posibilidades de experimentación.

- A través del juego, los educadores facilitarán los medios para favorecer el desarrollo del niño o la niña, generando sentimientos de afecto, amistad, compañerismo, ternura $\mathrm{y}$, en general, contribuyendo a una mayor sensibilidad con los otros, lo cual es fundamental para otra serie de actividades, como la observación, captación, comprensión de estímulos, etc., que crean en los niños y las niñas una mayor independencia y autonomía. 


\section{Formación profesional para atender la población infantil menor de tres años}

Considerando la importancia de brindar una atención pedagógica de calidad a los niños y niñas menores de tres años, surge la necesidad de que el docente o la docente de Educación Inicial se capacite para responder adecuadamente a las características de desarrollo y a las necesidades educativas de esta población.

Hasta hace pocos años la formación de estos profesionales, al menos en la Universidad de Costa Rica, estuvo centrada en el trabajo con los niños de edades entre cinco y seis años, es decir del Ciclo de Transición, según la denominación planteada por el Ministerio de Educación Pública. No obstante, tomando en cuenta las demandas de los últimos tiempos, así como la concepción de educación inicial, la cual abarca los primeros seis años de vida, se consideró necesario incorporar procesos de formación específicos, tanto para atender a los niños y niñas con edades entre cuatro y cinco años (Grupo Interactivo II, según Ministerio de Educación Pública) como para la atención del infante menor de tres años.

Es así como a partir de 1997 en el nivel de Bachillerato de la Carrera de Educación Preescolar que ofrece la Universidad de Costa Rica se divide el curso de Didáctica para la Educación Inicial en dos, en el primero se aborda el proceso específico para la intervención pedagógica con niños y niñas entre cero meses y tres años; y en el segundo se aborda el proceso para trabajar con niños y niñas entre cuatro y seis años. También complementariamente a partir de ese mismo año se incorpora el curso denominado Seminario de Práctica y Observación, en el cual las y los estudiantes realizan procesos de observación y práctica docente con infantes menores de tres años.

La experiencia práctica se ha desarrollado en diferentes instituciones y bajo diferentes modalidades. Inicialmente se realizó en los Centros Infantiles del Instituto Nacional de Seguros y de Recope. Luego se desarrolló en algunos EBAIS (Equipos Básicos de Atención Integral en Salud de la Caja Costarricense de Seguro Social). Posteriormente se hizo en la Sección de Educación Preescolar de la Universidad de Costa Rica. Y durante el año 2005, este proceso se realizó en la Casa Infantil Universitaria también de la Universidad de Costa Rica. 
Para desarrollar esta experiencia de intervención pedagógica con niños y niñas menores de tres años las estudiantes generalmente trabajan en parejas y siempre bajo la guía de una profesora supervisora. Cada pareja atiende dos infantes, uno con edad entre cero meses y un año y el otro con edad entre uno y tres años. Después de una valoración diagnóstica, las estudiantes plantean un plan de intervención para trabajar con cada niño o niña, el cual desarrollan aproximadamente durante seis semanas, trabajando una sesión semanal de dos horas, una hora trabajan con el niño o la niña menor de un año, y la otra con el niño o la niña con edad entre uno y tres años.

Para cada sesión de trabajo las estudiantes diseñan un planeamiento didáctico para desarrollar con cada niño o niña. Este planeamiento es comentado y revisado previamente con la profesora supervisora.

Por último, al finalizar el proceso las estudiantes preparan un informe descriptivo de cada niño o niña, donde comentan el proceso de trabajo desarrollado destacando los logros evidenciados y planteando las recomendaciones que consideren oportunas para continuar fortaleciendo el desarrollo integral del infante. De este informe se entrega una copia a la institución y otra a la familia.

En relación con el planeamiento didáctico, cabe señalar que el mismo se ha organizado utilizando como técnica de formulación de objetivos las tareas de desarrollo, las cuales según Peralta (1996, p. 106) fueron planteadas por R. Havighurst y utilizadas por P. Miller.

Las tareas de desarrollo "enuncian un aspecto clave e integrador del desarrollo, que toda persona debería resolver o superar satisfactoriamente en ciertos períodos de la vida, respondiendo así a sus propias necesidades, como a las de la cultura en que participa". Peralta (1996, p. 106). Esta misma autora señala como características de este tipo de objetivo las siguientes:

$\downarrow$ Es un objetivo desde el punto de vista del niño o niña.

- Hay un encadenamiento entre una tarea de desarrollo y las que siguen, ya que en la medida en que se van resolviendo adecuadamente se facilita la que continua.

- Este tipo de objetivo corresponde a una posición de educación permanente. 
- Requieren para su logro de múltiples y variadas situaciones de estimulación.

- Por su carácter integrador, es especialmente útil en Sala Cuna.

Fonseca (Cerdas, 2005, comunicación personal) por su parte, plantea que las tareas de desarrollo constituyen una habilidad o condición que debe lograr el ser humano en un momento determinado de su proceso de desarrollo. Tienen un carácter general e implican un proceso largo y continuo que pasa por diferentes etapas, las cuales se denominan subtareas.

Por ejemplo una tarea de desarrollo que debe lograr un infante con edad entre cero meses y dos años de edad es la Adquisición de la Marcha (caminar), para lograr esta habilidad el niño o niña pasa por las siguientes etapas o subtareas:

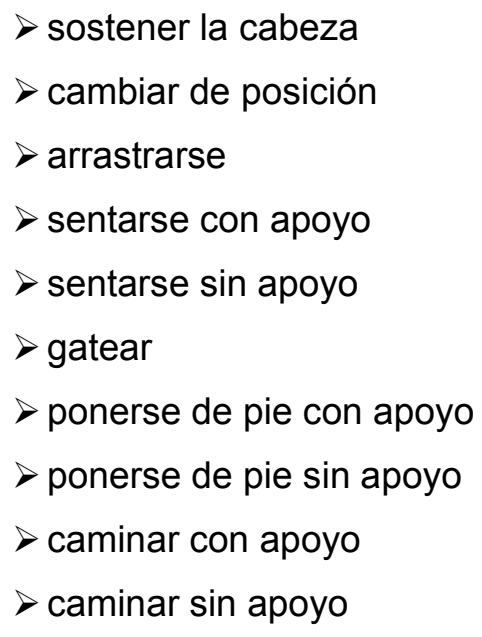

En la experiencia de intervención pedagógica que desarrollan los y las estudiantes de la carrera de Bachillerato y Licenciatura en Educación Preescolar de la Universidad de Costa Rica, ellos y ellas determinan, a partir del diagnóstico inicial que se le hace a cada niño o niña, las tareas de desarrollo y las subtareas que deben trabajarse para favorecer su desarrollo integral, según la edad y la etapa en que se encuentra; considerando los ámbitos socioafectivo, motor y cognitivo-lingüístico.

Asimismo, para cada subtarea propuesta, plantean una serie de actividades que favorezcan su alcance y preparan el material necesario para su ejecución. Cada proceso se 
cierra con la evaluación respectiva, utilizando en la mayoría de los casos como estrategia la observación y como instrumento el registro descriptivo o de observación.

\section{Experiencia del proceso de práctica docente con niños y niñas menores de tres años realizado en la Casa Infantil Universitaria}

Como ya se mencionó, durante el año 2005 el proceso de intervención pedagógica con niños y niñas menores de tres años, que se desarrolla en el marco del curso denominado Seminario de Práctica y Observación, de la carrera de Bachillerato y Licenciatura de la Universidad de Costa Rica, se realizó en la Casa Infantil Universitaria, durante el primer semestre del año. Se trabajó con un total de 24 niños y niñas con edades entre dos meses y tres años.

Esta experiencia resultó muy enriquecedora tanto para los niños y niñas, como para las estudiantes, las familias de los infantes y el personal de la Casa Infantil. A continuación se resumen las apreciaciones de algunas estudiantes, de algunas familias y del personal de la Casa Infantil Universitaria en relación con esta experiencia.

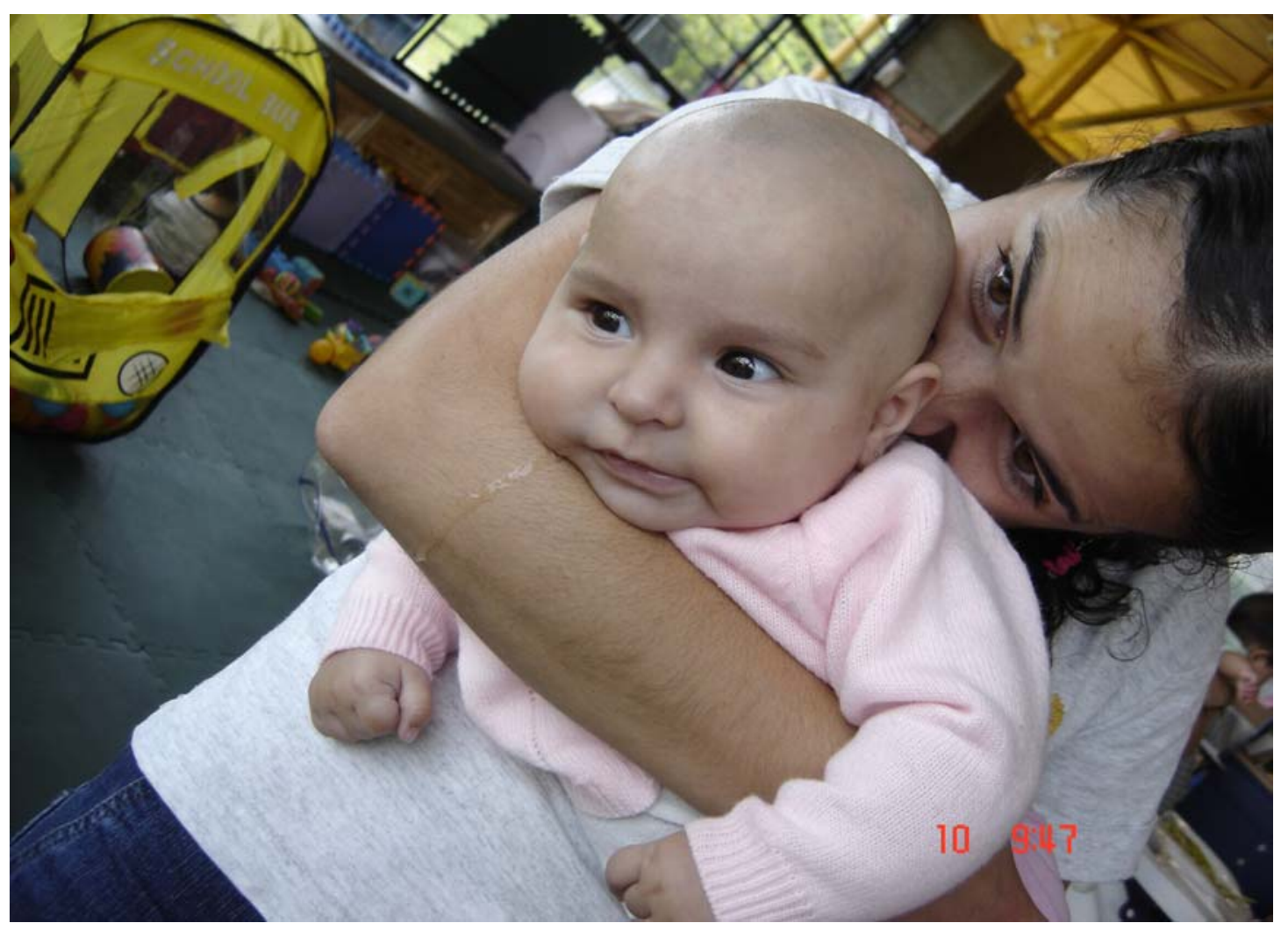


Dichas apreciaciones fueron recopiladas por medio de un album de registro personal que llevó cada estudiante y a través de una encuesta que se pasó a cada familia participante y al personal que laboraba en ese momento en la Casa Infantil.

Las estudiantes consideran que esta experiencia fortaleció su formación profesional de la siguiente manera:

Al aplicar los conocimientos adquiridos a lo largo de la carrera, así como darme cuenta que no es tan fácil el trabajo con bebés, pues hay que tomar en cuenta su edad, su etapa de desarrollo, sus necesidades e intereses. Fue una gran experiencia y me encantó... (Silvia Acuña Ortega)

Demasiado: pensando, corriendo, inventando y creando los MARAVILLOSOS planeamientos. Pero en fin esto me enseñó demasiado, viviendo experiencias reales que me permiten entender y aprender la verdadera realidad profesional. (María de los Angeles Rojas Lizano)

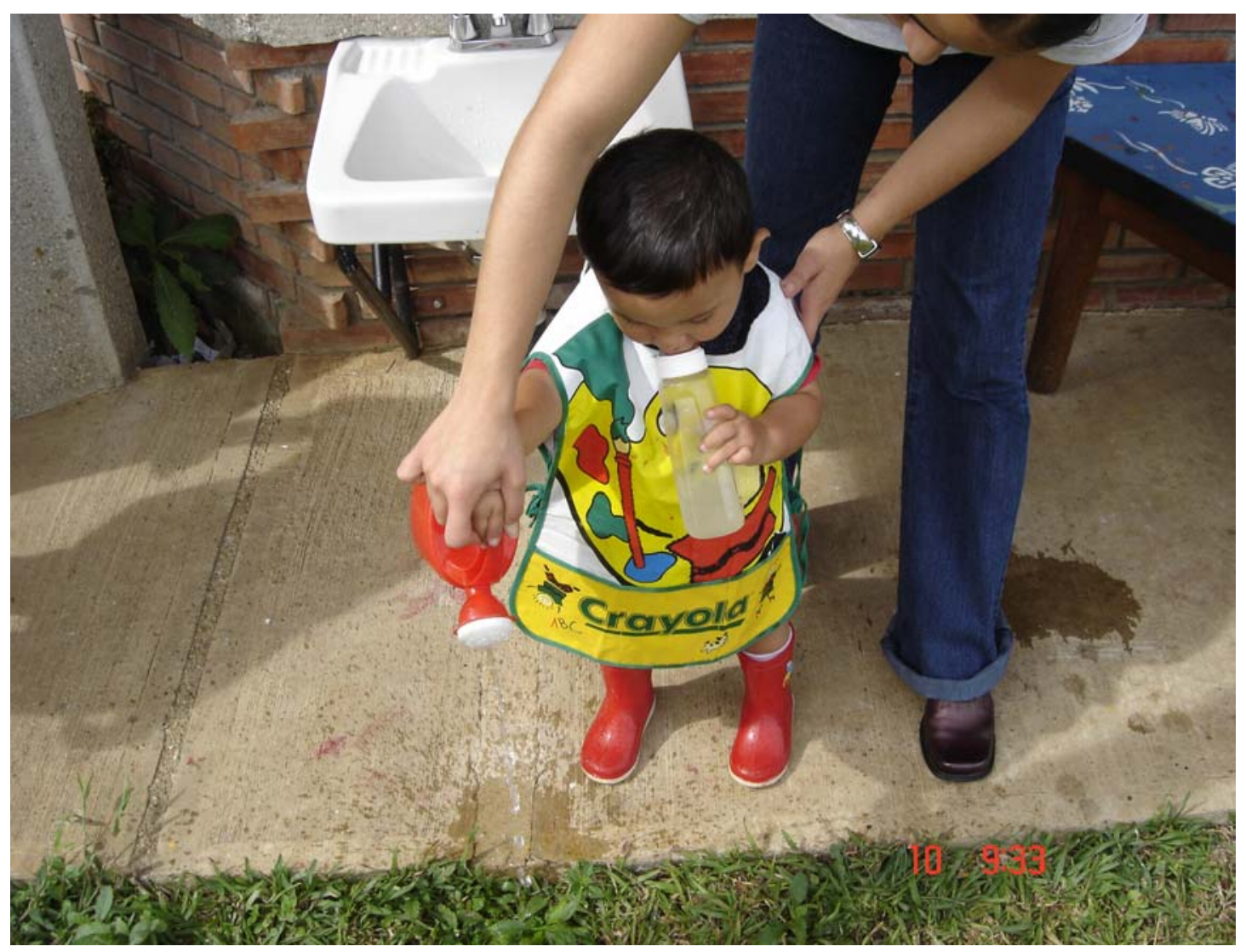


"Aprendiendo a planear, corrigiendo mis errores para obtener algo mejor, buscando la mejor manera para dirigirme a los niños" (María José Morales Ross)

"Es uno de los primeros acercamientos que tengo como docente a un niño o niña. Puse a prueba mi creatividad, pues la necesité al trabajar con ellos" (Carolina Blanco Calderón)

En cuanto a las apreciaciones que tienen los y las estudiantes madres o padres de familia de los niños y las niñas participantes respecto de los aportes que brindó la experiencia que realizaron las estudiantes junto con sus docentes de práctica, al aplicar un plan de atención y estimulación con su hijo o hija, se destacan las siguientes:

- Les permitió adquirir una guía de actividades para realizar con sus hijos, de manera que puedan contribuir al desarrollo integral de acuerdo a la edad del niño o niña.

- Las recomendaciones brindadas por las estudiantes fueron muy acertadas, así como el cariño brindado a cada niño y niña. Asimismo las actividades desarrolladas fueron atractivas para los niños y niñas, por ejemplo cuando se estimulaba la motora con materiales como esponjas y agua; y por medio de pintura con las manos.

- Una madre manifestó que al empezar a trabajar con su hija desde los 6 meses, se logró estimular tempranamente todas sus capacidades motoras, sociales, de lenguaje y en general todos los aspectos; lo que se ve reflejado ahora que tiene 1 año y 4 meses, tanto en su personalidad, como en su forma de hacer las cosas.

- Finalmente algunas madres mencionan que este tipo de trabajo les permitió conocer de manera más clara cuáles son las habilidades que sus hijos estaban desarrollando, conociendo en cuáles estaban bien, o por el contrario en cuáles estaban un poco atrasados o adelantados. Expresan que fue una bonita experiencia porque les demuestra o permite ver cualidades que no habían considerado dentro del desarrollo de su hijo o hija. 
Algunas sugerencias o recomendaciones que dan las familias para fortalecer esta experiencia son las siguientes:

$\checkmark$ Darle continuidad a la experiencia, ya que el proceso de aprendizaje tanto para el niño, como para los padres evoluciona con el transcurso del tiempo.

$\checkmark$ Sería importante que se le enseñara a los padres y madres qué cosas son buenas hacer y cuáles no, para estimular al niño, cuánto tiempo y otros aspectos relacionados con este tema.

$\checkmark$ Mantener la cercanía con los padres, pues esto da confianza en el trabajo realizado.

Por último, el personal que labora en la Casa Infantil Universitaria manifiesta lo siguiente en relación con la experiencia:

- El trabajo realizado por las estudiantes de Educación Preescolar es muy bueno, de gran valor y de mucho provecho tanto para el niño o la niña, con el que trabajaron, así como para los padres y madres de los menores, ya que con experiencias como esta se fomenta la educación integral de todas y todos.

- Las estudiantes y sus supervisoras manifestaron interés y empeño al trabajar durante un semestre de manera semanal con cada niño y niña, ofreciendo una atención de calidad y aportando material didáctico muy gustado por los niños y las niñas, permitiendo además que se convirtiera en un recurso muy valioso para la CIU.

- La observación realizada para cada niño y niña en sus diferentes áreas de desarrollo, permitió evaluar la conducta de cada uno según su edad; dando pautas a los padres y madres o encargados de los y las menores para fortalecer aquellas áreas que más lo necesitan. Además el aporte de las estudiantes fue valioso ya que colaboraron con el trabajo que se realiza en la CIU, realizando diagnósticos de algunos de los niños y las niñas, con lo cual pudieron corroborar sus criterios, al tener una segunda opinión.

- Las estudiantes practicantes a través de su trabajo, estimularon de manera muy positiva a la niñez de la CIU, ya que colaboraron en reforzar aspectos tales como lenguaje (ampliando vocabulario), destrezas motrices, entre otros; sirviendo de apoyo a lo que iba desarrollando el personal de la institución. 
- Esta experiencia es una forma de acercar a las estudiantes de preescolar al trabajo con niños y niñas de edades muy tempranas, siendo esto un factor muy importante para la población ya que es muy necesario que las estudiantes en formación conozcan y se familiaricen más con la niñez de 0 a 3 años.

- Por otra parte, consideran que se abrió un espacio para que la Sección de Preescolar de la Universidad de Costa Rica se vinculara aún más con la CIU, y de manera conjunta pudieran ofrecerles a las madres y a los padres estudiantes una alternativa más de atención y estimulación individualizada para sus hijos e hijas, acorde a las características y particularidades de cada uno.

- Finalmente, el personal de la CIU expresa que sería necesario que se continuaran llevando a cabo estas actividades de estimulación para que así los niños y las niñas continúen fortaleciendo sus capacidades tanto intelectuales, como motrices y socioafectivas, y de esta manera se contribuir con su desarrollo y crecimiento.

\section{Consideraciones finales}

- La población infantil menor de tres años requiere de una oferta educativa de calidad, que favorezca su desarrollo integral. De alguna manera estamos en deuda con estos niños y niñas, por lo tanto los adultos involucrados debemos asumir la responsabilidad correspondiente para ofrecerles alternativas tanto a ellos como a sus familias.

- La formación profesional que se brinda a los y las estudiantes de Educación Preescolar debe considerar la preparación para trabajar con esta población. Deben diseñarse experiencias concretas que les permitan a los futuros educadores y educadoras construir herramientas para abordar la labor pedagógica con este grupo de edad.

- La práctica supervisada realizada en la Casa Infantil Universitaria, constituye una posibilidad de las experiencias que podrían generarse para fortalecer los procesos de formación profesional que se requieren para atender a los niños y niñas menores de tres años. En este caso, los aportes fueron muy significativos para las diversas poblaciones involucradas: 
A los niños y las niñas participantes de las sesiones semanales, se les ofreció una atención oportuna de acuerdo a sus necesidades y requerimientos, permitiendo contribuir con la evolución de su crecimiento en las diversas áreas de desarrollo (motriz, lenguaje, cognitiva, socioemocional).

A las madres y padres estudiantes se les brindó apoyo y se les ofrecieron alternativas para la atención y estimulación de sus hijos e hijas, pues en la mayoría de los casos por ser primerizos y de poca edad tienen mayor cantidad de inquietudes.

$>$ Para las estudiantes representó una experiencia muy enriquecedora, pues muchas nunca habían tenido contacto con infantes tan pequeños, lo cual constituyó todo un reto tanto para planear las actividades como para ejecutarlas con los niños y las niñas.

> Para el personal de la Casa Infantil Universitaria resultó un apoyo significativo, ya que les aportó diagnósticos, actividades, recomendaciones, materiales didácticos, documentos de referencia, entre otros, que se pueden considerar cuando se trabaja con los y las pequeñas.

$>$ Las profesoras supervisoras, ampliamos nuestros conocimientos en relación con el manejo y atención de infantes menores de tres años, al observar la interacción y el desempeño de las estudiantes con los niños y niñas participantes. Asimismo tuvimos la oportunidad de aportar nuestra experiencia para contribuir con el proceso de formación de cada una de ellas.

\section{Referencias}

Asociación Mundial de Educadores Infantiles. (s.f.). La Educación de la Primera Infancia. Recuperado el 18 de abril de 2006, de http://www.waece.org/modelocentro/capitulo01.php

B. de Denies, E. Cristina. (1992). Didáctica del Nivel Inicial. Teoría y práctica de la enseñanza. Buenos Aires, Argentina: El Ateneo

Cerdas, Jeanneth. (2005, mayo). Comunicación Personal. Entrevista con Ana Fonseca Retana. Profesora Pensionada de la Escuela de Formación Docente, Carrera de Educación Preescolar. Universidad de Costa Rica. 
Cerdas González, Ana Isabel. (1995). Guía didáctica para el desarrollo y aprendizaje en niños de 0 a 3 años. San José, Costa Rica: Ministerio de Educación Pública, División de Desarrollo Curricular.

Feder, Valeria. (2001). Jardín Maternal y Desarrollo Emocional. Coordenadas para pensar las instituciones educativas que albergan niños pequeños. Revista Ensayos y Experiencias. 7(37): 11-23.

Gálvez, Inmaculada. (2000). La educación inicial en el ámbito internacional: Situación y perspectivas en Iberoamérica y en Europa. Recuperado el 15 de diciembre de 2005, de http://www.campus-oei.org/revista/rie22a06.htm

Ministerio de Educación Pública- Costa Rica. (2000). Programa de Estudio para el Ciclo Materno Infantil. San José, Costa Rica: MEP.

Peralta Espinosa, Victoria. (1996). El Currículo en el Jardín Infantil. Santiago, Chile: Editorial Andrés Bello.

Terré, Orlando. (s.f.). Estimulación y Desarrollo del Niño (0-3 años de vida). Guía práctica. [s.l.]: [s.n.]

Universidad de Costa Rica. (1996). Plan de Estudio: Carrera de Bachillerato y Licenciatura en Educación Preescolar. San José, Costa Rica: [s.n.] 